Probability, Networks and Algorithms

Probability, Networks and Algorithms
PNA The supremum of a Gaussian process over a random
interval

K.G. Dẹbicki, A.P. Zwart, S.C. Borst

Report PNA-R0218 July 31, 2002 
CWI is the National Research Institute for Mathematics and Computer Science. It is sponsored by the Netherlands Organization for Scientific Research (NWO).

CWI is a founding member of ERCIM, the European Research Consortium for Informatics and Mathematics.

CWI's research has a theme-oriented structure and is grouped into four clusters. Listed below are the names of the clusters and in parentheses their acronyms.

\section{Probability, Networks and Algorithms (PNA)}

Software Engineering (SEN)

Modelling, Analysis and Simulation (MAS)

Information Systems (INS)

Copyright (C) 2001, Stichting Centrum voor Wiskunde en Informatica

P.O. Box 94079, 1090 GB Amsterdam (NL)

Kruislaan 413, 1098 SJ Amsterdam (NL)

Telephone +31205929333

Telefax +31205924199

ISSN 1386-3711 


\title{
The Supremum of a Gaussian Process over a Random Interval
}

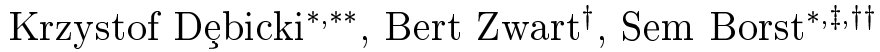 \\ ${ }^{*} C W I$ \\ P.O. Box 94079, 1090 GB Amsterdam, The Netherlands \\ ** Mathematical Institute, University of Wroctaw \\ pl. Grunwaldzki 2/4, 50-384 Wroctaw, Poland \\ ${ }^{\dagger}$ INRIA \\ Projet $R A P$ \\ BP 105, 78153 Le Chesnay, France \\ ${ }^{\ddagger}$ Bell Laboratories, Lucent Technologies \\ P.O. Box 636, Murray Hill, NJ 07974, USA \\ ${ }^{\dagger}$ Department of Mathematics \& Computer Science \\ Eindhoven University of Technology \\ P.O. Box 513, 5600 MB Eindhoven, The Netherlands
}

\begin{abstract}
The paper is concerned with the supremum of a centered Gaussian process with stationary increments over a random interval. The main result provides the exact asymptotics of the tail of the supremum distribution in the case that the length of the interval is regularly varying. In addition, we obtain explicit lower and upper bounds for the prefactor.
\end{abstract}

2000 Mathematics Subject Classification: 60G15 (primary), 60F10, 60G70 (secondary). Keywords 85 Phrases: exact asymptotics, extremes, fractional Brownian motion, Gaussian process, regular variation.

Note: Work carried out under the CWI project P1201. K. Dȩbicki was also supported by KBN under grant 5 P03A 02120 (2001-2003). 


\section{Introduction}

Let $\{X(s): s \geq 0\}$ be a centered Gaussian process with stationary increments. A classical problem in the extreme-value theory of Gaussian processes is to determine the tail behavior of

$$
\mathbb{P}\left(\sup _{s \in[0, T]} X(s)>u\right),
$$

for some fixed $T>0$.

Since the exact form of (1.1) is only known for a few special cases, recent analysis has focused on finding the asymptotic behavior of (1.1) as $u \rightarrow \infty$. In particular, Theorem D.3 in [9] (see also Piterbarg \& Prisyazhnyuk [10]) immediately implies that if the variance function $\sigma_{X}^{2}(s)=\operatorname{Var}(X(s))$ of the process $X(s)$ is strictly increasing on $[0, T]$, then under some regularity conditions for $\sigma_{X}(s)$ and the covariance $\operatorname{Cov}(X(s), X(t))$,

$$
\mathbb{P}\left(\sup _{s \in[0, T]} X(s)>u\right) \sim \text { Const } u^{\beta} \Psi\left(u / \sigma_{X}(T)\right)
$$

as $u \rightarrow \infty$, where $\Psi(x)=\mathbb{P}(\mathcal{N}>x)$ denotes the tail distribution of a standard normal random variable $\mathcal{N}$. For further results on the asymptotic behavior of (1.1), see e.g. Berman [2].

In the present paper, we focus on the case where $T$ is a random variable, independent of $X(s)$. Besides extending the classical theory, our motivation comes from a problem encountered in obtaining asymptotics for some fluid models, see [5].

We show that the qualitative tail behavior of the supremum may crucially depend on the variability of $T$. In particular, we focus on the case where $T$ has a heavy (i.e. regularly varying) tail. In this case, it is essentially the randomness in $T$ that determines the asymptotics, which are no longer of the form (1.2).

It is illuminating to show what happens if we take $X(s)=B_{\alpha}(s)$, where $B_{\alpha}(s)$ is a fractional Brownian motion (FBM) with Hurst parameter $\alpha \in(0,1]$, i.e., a centered Gaussian process with stationary increments, continuous sample paths, $B_{\alpha}(0)=0$, and variance function $\operatorname{Var}\left(B_{\alpha}(t)\right)=t^{2 \alpha}$. In this case, we can use the self-similarity of FBM and a classical result of Breiman [6] (see also Cline \& Samorodnitsky [7]) to get the following result.

Proposition 1.1 Let $T$ be a nonnegative random variable with regularly varying tail distribution at $\infty$ with index $\nu>0$. Then

$$
\mathbb{P}\left(\sup _{s \in[0, T]} B_{\alpha}(s)>u\right)=\mathbb{P}\left(T^{\alpha} \sup _{s \in[0,1]} B_{\alpha}(s)>u\right) \sim \mathbb{E}\left(\sup _{s \in[0,1]} B_{\alpha}(s)\right)^{\nu / \alpha} \mathbb{P}\left(T>u^{\frac{1}{\alpha}}\right)
$$

as $u \rightarrow \infty$.

The main goal of this note is to show that the asymptotics presented in Proposition 1.1 essentially remain the same for a much larger class of Gaussian processes than FBM; this result is stated in Theorem 2.1. The proof of Theorem 2.1, presented in Section 4, relies on the fact that (under some regularity conditions), a properly scaled Gaussian process converges to FBM, see Lemma 4.2. Section 2 also provides lower and upper bounds for the prefactor $\mathbb{E}\left(\sup _{s \in[0,1]} B_{\alpha}(s)\right)^{\nu / \alpha}$. In Section 3 we apply Theorem 2.1 to the analysis of the class of Gaussian processes with stationary increments and differentiable sample paths a.s. 


\section{Main result}

Before stating our main result, we first introduce some notation.

Throughout the paper, $X(t)$ is a centered Gaussian process with stationary increments, a.s. continuous sample paths, $X(0)=0$, and such that the standard deviation function $\sigma_{X}(t)=\sqrt{\operatorname{Var}(X(t))}$ satisfies the conditions

C1 $\sigma_{X}(t) \in C([0, \infty))$ is ultimately strictly increasing;

C2 $\sigma_{X}(t)$ is regularly varying at $\infty$ with index $\alpha \in(0,1]$;

C3 $\sigma_{X}(t)$ is regularly varying at 0 with index $\lambda \in(0,1]$.

We denote the generalized inverse function to $\sigma_{X}(t)$ by $\sigma_{X}^{-1}(t)=\inf \left\{s: \sigma_{X}(s) \geq t\right\}$. Moreover, let $L(\cdot)$ denote a function slowly varying at $\infty$. We assume that $T$ is a nonnegative random variable with regularly varying tail distribution at $\infty$ with index $\nu>0$, i.e., $\mathbb{P}(T>x)=L(x) x^{-\nu}$. For conciseness, we write $T \in \mathbf{R V}(\nu)$.

For any two real functions $f(\cdot)$ and $g(\cdot)$, we use the notational convention $f(u) \sim g(u)$ as $u \rightarrow \infty$ to denote that $f(u)=g(u)(1+o(1))$ as $u \rightarrow \infty$.

Throughout the paper, we assume that $T$ and $X(t)$ are independent.

The following constant plays a crucial role

$$
\mathcal{E}(\alpha, \gamma)=\mathbb{E}\left(\sup _{s \in[0,1]} B_{\alpha}(s)\right)^{\gamma},
$$

where $\alpha \in(0,1], \gamma>0$.

Theorem 2.1 Let $T \in \mathbf{R V}(\nu)$. If $X(t)$ is a centered Gaussian process with stationary increments and variance function $\sigma_{X}^{2}(t)$ such that conditions $\mathbf{C 1 - C 3}$ are satisfied, then

$$
\mathbb{P}\left(\sup _{s \in[0, T]} X(s)>u\right) \sim \mathcal{E}(\alpha, \nu / \alpha) \mathbb{P}\left(T>\sigma_{X}^{-1}(u)\right) \text { as } u \rightarrow \infty .
$$

Proof

The complete proof is presented in Section 4.

Remark 2.1 Note that Theorem 2.1 implies that $\sup _{s \in[0, T]} X(s)$ is regularly varying at $\infty$ with index $\nu / \alpha$.

In the following proposition we calculate the exact value of the constant $\mathcal{E}(\alpha, \gamma)$ for two special cases of the parameter $\alpha$, that is for $\alpha=1 / 2$ and $\alpha=1$.

Proposition 2.1 If $\gamma>0$, then

(i) $\mathcal{E}(1 / 2, \gamma)=\frac{1}{\sqrt{\pi}} 2^{(2+\gamma) / 2} \Gamma\left(\frac{\gamma+1}{2}\right)$;

(ii) $\mathcal{E}(1, \gamma)=\frac{1}{\sqrt{\pi}} 2^{\gamma / 2} \Gamma\left(\frac{\gamma+1}{2}\right)$.

Proof

Let $\alpha=1 / 2$. Then $\mathbb{P}\left(\sup _{s \in[0,1]} B_{1 / 2}(s)>x\right)=2 \Psi(x)$, which combined with the fact that

$$
\mathcal{E}(1 / 2, \gamma)=\gamma \int_{0}^{\infty} x^{\gamma-1} \mathbb{P}\left(\sup _{s \in[0,1]} B_{1 / 2}(s)>x\right) d x
$$


completes the proof of (i).

In order to prove (ii), it is enough to note that $B_{1}(t)=\mathcal{N} t$, where $\mathcal{N}$ is a standard normal random variable. Thus $\mathbb{P}\left(\sup _{s \in[0,1]} B_{1}(s)>x\right)=\Psi(x)$. Hence, a similar argumentation as in the proof of (i) completes the proof.

In the following proposition we show that for every fixed $\gamma>0$ the function $\mathcal{E}(\cdot, \gamma)$ is decreasing on $[0,1]$.

Proposition 2.2 Let $\gamma>0$. If $0<\alpha_{1} \leq \alpha_{2} \leq 2$, then

$$
\mathcal{E}\left(\alpha_{1}, \gamma\right) \geq \mathcal{E}\left(\alpha_{2}, \gamma\right)
$$

\section{Proof}

Let $\alpha_{1}, \alpha_{2} \in(0,2]$ and $\alpha_{1} \leq \alpha_{2}$. Moreover, let $Y(t)=B_{\alpha_{1}}\left(t^{\frac{\alpha_{2}}{\alpha_{1}}}\right)$. Clearly, we have

$$
\mathbb{P}\left(\sup _{s \in[0,1]} B_{\alpha_{1}}(s)>x\right)=\mathbb{P}\left(\sup _{s \in[0,1]} Y(s)>x\right)
$$

for all $x \geq 0$. Additionally, $\operatorname{Var}(Y(t))=\operatorname{Var}\left(B_{\alpha_{2}}(t)\right)=t^{2 \alpha_{2}}$ for every $t \in[0,1]$ and

$$
\operatorname{Var}(Y(t)-Y(s))=\left|t^{\frac{\alpha_{2}}{\alpha_{1}}}-s^{\frac{\alpha_{2}}{\alpha_{1}}}\right|^{2 \alpha_{1}} \geq|t-s|^{2 \alpha_{2}}=\operatorname{Var}\left(B_{\alpha_{2}}(t)-B_{\alpha_{2}}(s)\right)
$$

for all $s, t \in[0,1]$.

Thus, using the Slepian inequality (e.g. Corollary 2.4 in [1]),

$$
\mathbb{P}\left(\sup _{s \in[0,1]} Y(s)>x\right) \geq \mathbb{P}\left(\sup _{s \in[0,1]} B_{\alpha_{2}}(s)>x\right)
$$

for all $x \geq 0$, which in view of (2.1) completes the proof.

Combining Proposition 2.1 with Proposition 2.2, we obtain the following bounds for $\mathcal{E}(\alpha, \gamma)$.

Corollary 2.1 Let $\gamma>0$.

(i) If $\alpha \in(0,1 / 2)$, then $\mathcal{E}(\alpha, \gamma) \geq \frac{1}{\sqrt{\pi}} 2^{(2+\gamma) / 2} \Gamma\left(\frac{\gamma+1}{2}\right)$.

(ii) If $\alpha \in(1 / 2,1)$, then $\frac{1}{\sqrt{\pi}} 2^{\gamma / 2} \Gamma\left(\frac{\gamma+1}{2}\right) \leq \mathcal{E}(\alpha, \gamma) \leq \frac{1}{\sqrt{\pi}} 2^{1+(\gamma / 2)} \Gamma\left(\frac{\gamma+1}{2}\right)$.

\section{Application to Gaussian integrated processes}

In this section we focus on the class of Gaussian processes with stationary increments that have, in contrast to the fractional Brownian motion analyzed in Proposition 1.1, differentiable sample paths a.s. In particular, we consider Gaussian processes $X(t)$ such that

$$
X(t)=\int_{0}^{t} Z(s) d s
$$

where $Z(t)$ is a centered stationary Gaussian process with continuous covariance function $R(t)=\operatorname{Cov}(Z(s+t), Z(s))$. 
Proposition 3.1 Let $T \in \mathbf{R V}(\nu)$ and $f_{R}(t)$ be the spectral density function of the covariance function $R(t)$ of a centered stationary Gaussian process $Z(t)$.

If $0<f_{R}(0)<\infty$ and $R(t)$ is continuous, then for $X(t)=\int_{0}^{t} Z(s) d s$,

$$
\mathbb{P}\left(\sup _{t \in[0, T]} X(t)>u\right) \sim \frac{1}{\sqrt{\pi}} 2^{1+\nu} \Gamma\left(\nu+\frac{1}{2}\right) \mathbb{P}\left(T>\sigma_{X}^{-1}(u)\right) \text { as } u \rightarrow \infty .
$$

Proof

The idea of the proof is to check that conditions C1-C3 hold and then use Theorem 2.1. Note that $\sigma_{X}^{2}(t)=2 \int_{0}^{t} d s \int_{0}^{s} R(v) d v$. Hence $\left(\sigma_{X}^{2}\right)^{\prime}(t)=2 \int_{0}^{t} R(v) d v$, which combined with the fact that

$$
\int_{0}^{\infty} R(t) d t=\pi f_{R}(0)
$$

immediately yields that $\mathbf{C 1}$ is satisfied.

Moreover, (3.1) implies that $\int_{0}^{t} R(v) d v$ is slowly varying at $\infty$. Hence, by Karamata's theorem, $\sigma_{X}^{2}(t)$ is regularly varying at $\infty$ with index 1 . Thus $\mathbf{C 2}$ holds with $\alpha=1 / 2$.

Since $\lim _{t \rightarrow 0} \frac{\sigma_{X}^{2}(t)}{t^{2}}=R(0)$, assumption C3 is satisfied with $\lambda=1$.

This completes the proof.

Proposition 3.2 Let $T \in \mathbf{R V}(\nu)$ and $Z(t)$ be a centered stationary process with continuous covariance function $R(t)$ such that $R(t)>0$ and is regularly varying at $\infty$ with index $2 \alpha-2$, for $\alpha \in(1 / 2,1)$. Then for $X(t)=\int_{0}^{t} Z(s) d s$,

$$
\mathbb{P}\left(\sup _{t \in[0, T]} X(t)>u\right) \sim \mathcal{E}(\alpha, \nu / \alpha) \mathbb{P}\left(T>\sigma_{X}^{-1}(u)\right) \text { as } u \rightarrow \infty .
$$

Proof

Since the proof is analogous to the proof of Proposition 3.1, we only note that due to Karamata's theorem $\sigma_{X}^{2}(t)=2 \int_{0}^{t} d s \int_{0}^{s} R(v) d v$ is regularly varying at $\infty$ with index $2 \alpha$. Hence following Theorem 2.1, the proof is completed.

\section{Proof of Theorem 2.1}

Before presenting the proof of Theorem 2.1, we first state some lemmas that may be of independent interest.

Our first result is a property for regularly varying functions which is a variation of Potter's theorem, see Theorem 1.5.6 in [4].

Lemma 4.1 If C2-C3 hold, then there exist $\beta, B>0$ such that for sufficiently large $u$ and all $x \in[0,1]$,

$$
\sigma_{X}(u x) \leq B x^{\beta} \sigma_{X}(u) .
$$

Proof

Take $\delta<\alpha$ and let $x^{*} \geq 1$ be such that Theorem 1.5.6 (iii) of [4] is satisfied. This theorem implies that there exists an $A$ such that, for $x>y \geq x^{*}$, we have

$$
\frac{\sigma_{X}(y)}{\sigma_{X}(x)} \leq A(y / x)^{\beta_{1}}
$$


with $\beta_{1}=\alpha-\delta$. Next, note that for $y<x^{*}$,

$$
\frac{\sigma_{X}(y)}{\sigma_{X}(x)}=\frac{\sigma_{X}(y)}{\sigma_{X}\left(x^{*}\right)} \frac{\sigma_{X}\left(x^{*}\right)}{\sigma_{X}(x)} \leq A^{\prime} y^{\beta_{2}} A\left(x^{*} / x\right)^{\beta_{1}},
$$

which follows from Theorem 1.5.6 (iii) of [4] and the fact that $\sigma_{X}(x)$ is regularly varying at 0 . Now, take $\beta=\min \left\{\beta_{1}, \beta_{2}\right\}$. Combining (4.2) with (4.3), we conclude that for $x>x^{*}$ and $y>0$, there exists a constant $B$ such that

$$
\frac{\sigma_{X}(y)}{\sigma_{X}(x)} \leq B(y / x)^{\beta}
$$

which implies the lemma.

The next lemma states the well-known fact that a suitably scaled version of $X(s)$ converges to FBM (see e.g Proposition 7.2.11 in [11] or [8]). We give a short proof under our specific assumptions.

For a given function $f(u)$ define $X_{f(u)}(s)=\frac{X(f(u) s)}{\sigma_{X}(f(u))}$.

Lemma 4.2 Let $X(t)$ be a centered Gaussian process with stationary increments such that C1-C3 are satisfied and let $f(u)>0$ be a function such that $\lim _{u \rightarrow \infty} f(u)=\infty$. Then the process $X_{f(u)}(s)$ weakly converges in $C([0,1])$, as $u \rightarrow \infty$, to $B_{\alpha}(s)$.

\section{Proof}

In order to prove Lemma 4.2, it suffices to show the convergence of appropriate finitedimensional distributions of $X_{f(u)}(s)$ as $u \rightarrow \infty$ and tightness.

Note that for every $u>0, X_{f(u)}(s)$ is a centered Gaussian stochastic process with stationary increments. Moreover, for every $s \in[0,1]$,

$$
\lim _{u \rightarrow \infty} \operatorname{Var}\left(X_{f(u)}(s)\right)=s^{2 \alpha},
$$

and

$$
\lim _{u \rightarrow \infty} \operatorname{Var}\left(X_{f(u)}\left(s_{1}\right)-X_{f(u)}\left(s_{2}\right)\right)=\lim _{u \rightarrow \infty} \operatorname{Var}\left(X_{f(u)}\left(s_{1}-s_{2}\right)\right)=\left|s_{1}-s_{2}\right|^{2 \alpha} .
$$

Hence, we conclude the convergence of all finite-dimensional distributions. In order to show tightness, it suffices to prove that for sufficiently large $u$

$$
\mathbb{E}\left|X_{f(u)}\left(s_{1}\right)-X_{f(u)}\left(s_{2}\right)\right|^{2} \leq C\left|s_{1}-s_{2}\right|^{\delta}
$$

for some $C, \delta>0$ and all $s_{1}, s_{2} \in[0,1]$ (see e.g. Theorem 12.3 in Billingsley [3]). Now note that

$$
\begin{aligned}
\mathbb{E}\left|X_{f(u)}\left(s_{1}\right)-X_{f(u)}\left(s_{2}\right)\right|^{2} & =\operatorname{Var}\left(X_{f(u)}\left(s_{1}-s_{2}\right)\right) \\
& =\left(\frac{\sigma_{X}\left(f(u)\left(s_{1}-s_{2}\right)\right)}{\sigma_{X}(f(u))}\right)^{2} \leq\left(B\left|s_{1}-s_{2}\right|^{\beta}\right)^{2},
\end{aligned}
$$

where the last inequality follows from Lemma 4.1. Thus, the proof is completed.

Let $X_{u, t}(s)=\frac{X\left(\sigma_{X}^{-1}(u) t s\right)}{\sigma_{X}\left(\sigma_{X}^{-1}(u) t\right)}$.

The following corollary will play a crucial role in the proof of Theorem 2.1.

Corollary 4.1 Let $\epsilon>0$ be such that $\lim _{u \rightarrow \infty} \sigma_{X}^{-1}(u) u^{-\epsilon}=\infty$. Then for every $t \in\left[u^{-\epsilon}, A\right]$, the process $X_{u, t}(s)$ weakly converges in $C([0,1])$, as $u \rightarrow \infty$, to $B_{\alpha}(s)$. 


\section{Proof}

The proof follows from Lemma 4.2 applied to $X_{u, t}(s)$ with $f(u)=\sigma_{X}^{-1}(u) t$.

We now state a final preparatory lemma.

Lemma 4.3 Let $X(t)$ be a centered Gaussian process with stationary increments such that conditions C1-C3 are satisfied. Then for every $\epsilon>0$ and $\gamma>0$,

$$
\mathbb{P}\left(\sup _{s \in\left[0, \sigma_{X}^{-1}(u) u^{-\epsilon}\right]} X(s)>u\right)=o\left(u^{-\gamma}\right) \text { as } u \rightarrow \infty .
$$

\section{Proof}

Since $\sigma_{X}^{2}(t)$ is regularly varying at 0 with index $2 \lambda$, there exists a $T>0$ such that $\sigma_{X}^{2}(t) \leq \sigma_{X}^{2}(1) t^{\lambda}$, and $\exp \left(-t^{\lambda}\right) \leq 1-\frac{t^{\lambda}}{2}$, for every $t \in[0, T]$. Moreover, let $k_{0} \in \mathbb{N}$ be such that $\sigma_{X}^{2}(t) \geq \sigma_{X}^{2}(1)$ for all $t \geq k_{0} T$.

Now, for sufficiently large $u>0$,

$$
\begin{aligned}
& \mathbb{P}\left(\sup _{s \in\left[0, \sigma_{X}^{-1}(u) u^{-\epsilon}\right]} X(s)>u\right) \leq \\
& \leq \mathbb{P}\left(\sup _{s \in\left[0, k_{0} T\right]} X(s)>u\right)+\sum_{k=k_{0}}^{\left[\frac{\sigma_{X}^{-1}(u) u^{-\epsilon}}{T}\right]} \mathbb{P}\left(\sup _{s \in[k T,(k+1) T]} X(s)>u\right) \\
& \leq \mathbb{P}\left(\sup _{s \in\left[0, k_{0} T\right]} X(s)>u\right)+\sum_{k=k_{0}}^{\left[\frac{\sigma_{X}^{-1}(u) u^{-\epsilon}}{T}\right]} \mathbb{P}\left(\sup _{s \in[k T,(k+1) T]} \frac{X(s)}{\sigma_{X}(s)}>\frac{u}{\sigma_{X}\left(\sigma_{X}^{-1}(u) u^{-\epsilon}\right)}\right) \\
& \leq \mathbb{P}\left(\sup _{s \in\left[0, k_{0} T\right]} X(s)>u\right)+\sum_{k=k_{0}}^{\left[\frac{\sigma_{X}^{-1}(u) u^{-\epsilon}}{T}\right]} \mathbb{P}\left(\sup _{s \in[k T,(k+1) T]} \frac{X(s)}{\sigma_{X}(s)}>u^{\epsilon \alpha / 2}\right),
\end{aligned}
$$

where the last inequality follows from the fact that $u / \sigma_{X}\left(\sigma_{X}^{-1}(u) u^{-\epsilon}\right)$ is regularly varying with index $\epsilon \alpha$.

Due to the Borell inequality (see Theorem 2.1 in [1]), the first term in (4.4) may be bounded by

$$
\mathbb{P}\left(\sup _{t \in\left[0, k_{0} T\right]} X(t)>u\right) \leq 2 \exp \left(-\frac{\left(u-\mathbb{E} \sup _{t \in\left[0, k_{0} T\right]} X(t)\right)^{2}}{2}\right) \text { as } u \rightarrow \infty .
$$

Thus it suffices to find a bound for the sum in (4.4).

Let $Z(t)$ be a centered stationary Gaussian process with covariance function

$$
\operatorname{Cov}(Z(s+t), Z(s))=\exp \left(-t^{\lambda}\right) .
$$

Note that for every $s, t \in[k T,(k+1) T]\left(k \geq k_{0}\right)$,

$$
\begin{aligned}
\operatorname{Cov} & \left(\frac{X(t)}{\sigma_{X}(t)}, \frac{X(s)}{\sigma_{X}(s)}\right)=\frac{\sigma_{X}^{2}(t)+\sigma_{X}^{2}(s)-\sigma_{X}^{2}(|t-s|)}{2 \sigma_{X}(t) \sigma_{X}(s)} \geq \\
& \geq 1-\frac{\sigma_{X}^{2}(|t-s|)}{2 \sigma_{X}(t) \sigma_{X}(s)} \geq 1-\frac{\sigma_{X}^{2}(|t-s|)}{2 \sigma_{X}^{2}(1)} \\
& \geq 1-\frac{|t-s|^{\lambda}}{2} \\
& \geq \exp \left(-|t-s|^{\lambda}\right)=\operatorname{Cov}(Z(s), Z(t)) .
\end{aligned}
$$


Thus for every $k \geq k_{0}$ and sufficiently large $u$,

$$
\begin{aligned}
\mathbb{P}\left(\sup _{t \in[k T,(k+1) T]} \frac{X(t)}{\sigma_{X}(t)}>u^{\epsilon \alpha / 2}\right) & \leq \mathbb{P}\left(\sup _{t \in[0, T]} Z(t)>u^{\epsilon \alpha / 2}\right) \\
& \leq 2 \exp \left(\frac{-\left(u^{\epsilon \alpha / 2}-\mathbb{E}\left(\sup _{t \in[0, T]} Z(t)\right)\right)^{2}}{2}\right)
\end{aligned}
$$

where (4.5) follows from the Slepian inequality (see Theorem C.1 in Piterbarg [9]) and (4.6) is due to the Borell inequality. Combining (4.6) with (4.4), we obtain

$$
\begin{aligned}
& {\left[\frac{\sigma_{X}^{-1}(u) u^{-\epsilon}}{T}\right] \mathbb{P}\left(\sup _{t \in[k T,(k+1) T]} \frac{X(t)}{\sigma_{X}(t)}>u^{\epsilon \alpha / 2}\right) \leq} \\
& \leq 2\left[\frac{\sigma_{X}^{-1}(u) u^{-\epsilon}}{T}\right] \exp \left(-\frac{\left(u^{\epsilon \alpha / 2}-\mathbb{E}\left(\sup _{t \in[0, T]} Z(t)\right)\right)^{2}}{2}\right)=o\left(u^{-\gamma}\right)
\end{aligned}
$$

as $u \rightarrow \infty$ for every $\gamma>0$. This completes the proof.

Proof of Theorem 2.1

Let $G(x)=\mathbb{P}(T \leq x)$ be the distribution function of $T$. Let $a, A>0$ be given and $\epsilon>0$ be such that $\sigma_{X}^{-1}(u) u^{-\epsilon} \rightarrow \infty$ as $u \rightarrow \infty$. We have, with $u^{-\epsilon} \leq a \leq A$,

$$
\begin{aligned}
\mathbb{P}\left(\sup _{s \in[0, T]} X(s)>u\right)= & \int_{0}^{\infty} \mathbb{P}\left(\sup _{s \in[0, t]} X(s)>u\right) d G(t) \\
= & \int_{0}^{\infty} \mathbb{P}\left(\sup _{s \in\left[0, \sigma_{X}^{-1}(u) t\right]} X(s)>u\right) d G\left(\sigma_{X}^{-1}(u) t\right) \\
= & \int_{0}^{u^{-\epsilon}} \mathbb{P}\left(\sup _{s \in\left[0, \sigma_{X}^{-1}(u) t\right]} X(s)>u\right) d G\left(\sigma_{X}^{-1}(u) t\right) \\
& +\int_{u^{-\epsilon}}^{a} \mathbb{P}\left(\sup _{s \in\left[0, \sigma_{X}^{-1}(u) t\right]} X(s)>u\right) d G\left(\sigma_{X}^{-1}(u) t\right) \\
& +\int_{a}^{A} \mathbb{P}\left(\sup _{s \in\left[0, \sigma_{X}^{-1}(u) t\right]} X(s)>u\right) d G\left(\sigma_{X}^{-1}(u) t\right) \\
& +\int_{A}^{\infty} \mathbb{P}\left(\sup _{s \in\left[0, \sigma_{X}^{-1}(u) t\right]} X(s)>u\right) d G\left(\sigma_{X}^{-1}(u) t\right) \\
= & I_{1}+I_{2}+I_{3}+I_{4} .
\end{aligned}
$$

We analyze each of the integrals $I_{1}, I_{2}, I_{3}, I_{4}$ separately.

\section{Integral $I_{1}$}

According to Lemma 4.3, we have

$$
\begin{aligned}
\int_{0}^{u^{-\epsilon}} \mathbb{P}\left(\sup _{s \in\left[0, \sigma_{X}^{-1}(u) t\right]} X(s)>u\right) d G\left(\sigma_{X}^{-1}(u) t\right) & \leq \mathbb{P}\left(\sup _{s \in\left[0, \sigma_{X}^{-1}(u) u^{-\epsilon}\right]} X(s)>u\right) \\
& =o\left(\mathbb{P}\left(T>\sigma_{X}^{-1}(u)\right)\right) \text { as } u \rightarrow \infty .
\end{aligned}
$$




\section{Integral $I_{2}$}

According to (4.1), we have $\sigma_{X}\left(\sigma_{X}^{-1}(u) t\right) \leq B t^{\beta} u$ and thus

$$
\frac{u}{\sigma_{X}\left(\sigma_{X}^{-1}(u) t\right)} \geq \frac{1}{B t^{\beta}}
$$

Hence,

$$
\begin{aligned}
I_{2} & =\int_{u^{-\epsilon}}^{a} \mathbb{P}\left(\sup _{s \in\left[0, \sigma_{X}^{-1}(u) t\right]} X(s)>u\right) d G\left(\sigma_{X}^{-1}(u) t\right) \\
& \leq \int_{u^{-\epsilon}}^{a} \mathbb{P}\left(\sup _{s \in[0,1]} \frac{X\left(\sigma_{X}^{-1}(u) t s\right)}{\sigma_{X}\left(\sigma_{X}^{-1}(u) t\right)}>\frac{u}{\sigma_{X}\left(\sigma_{X}^{-1}(u) t\right)}\right) d G\left(\sigma_{X}^{-1}(u) t\right) \\
& \leq \int_{u^{-\epsilon}}^{a} \mathbb{P}\left(\sup _{s \in[0,1]} X_{u, t}(s)>\frac{1}{B t^{\beta}}\right) d G\left(\sigma_{X}^{-1}(u) t\right) .
\end{aligned}
$$

Note that according to Lemma 4.2 , as $u \rightarrow \infty$, the process $X_{u, t}(s)$ weakly converges to $B_{\alpha}(s)$ in $C([0,1])$ for $t \in\left[u^{-\epsilon}, a\right]$. Since the functional $\sup _{s \in[0,1]} f(s)$ is continuous in the uniform metric, there exists a constant $C_{0}>0$ such that

$$
\mathbb{P}\left(\sup _{s \in[0,1]} X_{u, t}(s)>C_{0}\right) \leq 1 / 2
$$

for sufficiently large $u$ and $t \in\left[u^{-\epsilon}, a\right]$. Hence, from the Borell inequality (see e.g. Theorem D.1 in [9])

$$
\mathbb{P}\left(\sup _{s \in[0,1]} X_{u, t}(s)>x\right) \leq 2 \Psi\left(x-C_{0}\right)
$$

for every $x$. Combining (4.8) with (4.9),

$$
\begin{aligned}
\int_{u^{-\epsilon}}^{a} \mathbb{P}\left(\sup _{s \in[0,1]} X_{u, t}(s)>\frac{1}{B t^{\beta}}\right) d G\left(\sigma_{X}^{-1}(u) t\right) & \leq 2 \int_{u^{-\epsilon}}^{a} \Psi\left(\left(t^{-\beta} / B\right)-C_{0}\right) d G\left(\sigma_{X}^{-1}(u) t\right) \\
& \leq 2 \int_{u^{-\epsilon}}^{a} \Psi\left(t^{-\beta / 2}\right) d G\left(\sigma_{X}^{-1}(u) t\right)
\end{aligned}
$$

for sufficiently large $u$. Now, using integration by parts, we have

$$
\begin{aligned}
& 2 \int_{u^{-\epsilon}}^{a} \Psi\left(t^{-\beta / 2}\right) d G\left(\sigma_{X}^{-1}(u) t\right)=-2 \int_{u^{-\epsilon}}^{a} \Psi\left(t^{-\beta / 2}\right) d \bar{G}\left(\sigma_{X}^{-1}(u) t\right) \\
& \quad=2 \Psi\left(u^{\epsilon \beta / 2}\right) \bar{G}\left(\sigma_{X}^{-1}(u) u^{-\epsilon}\right)-2 \Psi\left(a^{-\beta / 2}\right) \bar{G}\left(\sigma_{X}^{-1}(u) a\right)+2 \int_{u^{-\epsilon}}^{a} \bar{G}\left(\sigma_{X}^{-1}(u) t\right) d \Psi\left(t^{-\beta / 2}\right) \\
& \quad \leq 2 \Psi\left(u^{\epsilon \beta / 2}\right)+2 \int_{u^{-\epsilon}}^{a} \bar{G}\left(\sigma_{X}^{-1}(u) t\right) d \Psi\left(t^{-\beta / 2}\right) \\
& \left.\quad \leq 2 \Psi\left(u^{\epsilon \beta / 2}\right)+2 \bar{G}\left(\sigma_{X}^{-1}(u)\right)\right) \int_{u^{-\epsilon}}^{a} t^{-\nu-\mu} d \Psi\left(t^{-\beta / 2}\right)
\end{aligned}
$$

where (4.11) follows from Potter's theorem and the fact that $\mu>0$. Moreover,

$$
\begin{aligned}
\int_{u^{-\epsilon}}^{a} t^{-\nu-\mu} d \Psi\left(t^{-\beta / 2}\right) & =\frac{\beta}{2 \sqrt{2 \pi}} \int_{u^{-\epsilon}}^{a} t^{-\nu-\mu} t^{-(\beta / 2)-1} \exp \left(-\frac{t^{-\beta}}{2}\right) d t \\
& =\frac{1}{\sqrt{2 \pi}} \int_{a^{-1}}^{u^{\epsilon}} y^{v+\mu+\beta / 2-1} \exp \left(-\frac{y^{\beta}}{2}\right) d y \\
& =o(1)
\end{aligned}
$$


as $u \rightarrow \infty$ and $a \rightarrow \infty$, where (4.12) follows from the change of variable $y \equiv 1 / t$.

Thus, combining (4.8) with (4.10), (4.11) and (4.13), we conclude that $I_{2}=o\left(\bar{G}\left(\sigma_{X}^{-1}(u)\right)\right)$ as $u \rightarrow \infty$.

\section{Integral $I_{3}$}

We have

$$
\begin{aligned}
I_{3} & =\int_{a}^{A} \mathbb{P}\left(\sup _{s \in\left[0, \sigma_{X}^{-1}(u) t\right]} X(s)>u\right) d G\left(\sigma_{X}^{-1}(u) t\right) \\
& =\int_{a}^{A} \mathbb{P}\left(\sup _{s \in[0,1]} X_{u, t}(s)>\frac{u}{\sigma_{X}\left(\sigma_{X}^{-1}(u) t\right)}\right) d G\left(\sigma_{X}^{-1}(u) t\right) .
\end{aligned}
$$

According to Theorem 1.5.2 in [4],

$$
\frac{u}{\sigma_{X}\left(\sigma_{X}^{-1}(u) t\right)} \rightarrow t^{-H} \text { as } u \rightarrow \infty
$$

uniformly for $t \in[a, A]$. Moreover, according to Lemma 4.2, the process $X_{u, t}(s)$, converges weakly in $C([0,1])$ to $B_{\alpha}(s)$, uniformly in $t \in[a, A]$. Hence, for sufficiently large $u$,

$$
\begin{aligned}
I_{3} & \sim \int_{a}^{A} \mathbb{P}\left(\sup _{s \in[0,1]} B_{\alpha}(s)>t^{-\alpha}\right) d G\left(\sigma_{X}^{-1}(u) t\right) \\
& =\mathbb{P}\left(\left(\sup _{s \in[0,1]} B_{\alpha}(s)\right)^{1 / H} T>\sigma^{-1}(u) ; a \sigma_{X}^{-1}(u) \leq T \leq A \sigma_{X}^{-1}(u)\right) .
\end{aligned}
$$

\section{Integral $I_{4}$}

We have

$$
\int_{A}^{\infty} \mathbb{P}\left(\sup _{s \in\left[0, \sigma_{X}^{-1}(u) t\right]} X(s)>u\right) d G\left(\sigma_{X}^{-1}(u) t\right) \leq \bar{G}\left(A \sigma_{X}^{-1}(u)\right) \sim A^{-\nu} \bar{G}\left(\sigma_{X}^{-1}(u)\right) \text { as } u \rightarrow \infty .
$$

Now letting $a \rightarrow 0$ and $A \rightarrow \infty$, we obtain that $I_{1}+I_{2}+I_{4}=o\left(\bar{G}\left(\sigma_{X}^{-1}(u)\right)\right)$. Thus the main contribution to the asymptotics comes from $I_{3}$. In order to complete the proof, we need to show that

$$
\lim _{a \downarrow 0, A \rightarrow \infty} \liminf _{u \rightarrow \infty} \frac{I_{3}}{\bar{G}\left(\sigma_{X}^{-1}(u)\right)}=\mathbb{E}\left(\left(\sup _{s \in[0,1]} B_{\alpha}(s)\right)^{\nu / H}\right) .
$$

(The limsup is trivial: take $a=0, A=\infty$, and use the computation in the Introduction.) Define $\bar{B}=\left(\sup _{s \in[0,1]} B_{\alpha}(s)\right)^{1 / H}$. Note that $I_{3}$ is asymptotically larger than

$$
\mathbb{P}\left(\bar{B} T>\sigma_{X}^{-1}(u)\right)-\mathbb{P}\left(\bar{B} T>\sigma_{X}^{-1}(u) ; T \leq a \sigma_{X}^{-1}(u)\right)-\mathbb{P}\left(T \geq A \sigma_{X}^{-1}(u)\right) .
$$

The last term can be handled in a similar fashion as $I_{4}$. This leaves us with showing that

$$
\lim _{a \downarrow 0} \limsup _{u \rightarrow \infty} \frac{\mathbb{P}\left(\bar{B} T>\sigma_{X}^{-1}(u) ; T \leq a \sigma_{X}^{-1}(u)\right)}{\mathbb{P}\left(T>\sigma_{X}^{-1}(u)\right)}=0 .
$$

Set $x=\sigma_{X}^{-1}(u)$ and write, using Markov's inequality and applying partial integration,

$$
\begin{aligned}
\mathbb{P}(\bar{B} T>x ; T<a x) & =\int_{0}^{a x} d G(t) \mathbb{P}(\bar{B}>x / t) \leq \\
& \leq \int_{0}^{a x} d G(t) C(x / t)^{-\nu-\epsilon}=-\int_{0}^{a x} C(x / t)^{-\nu-\epsilon} d \bar{G}(t) \\
& =C \mathbb{P}(T>a x) a^{\nu+\epsilon}+(\nu+\epsilon) C x^{-\nu-\epsilon} \int_{0}^{a x} \bar{G}(t) t^{\nu+\epsilon-1} d t \\
& \sim C \mathbb{P}(T>a x) a^{\nu+\epsilon}+C^{\prime} L(a x) x^{-\nu} a^{\epsilon}
\end{aligned}
$$


as $x \rightarrow \infty$, where $C, C^{\prime}$ are finite constants independent of $a$. The last equivalence follows from Karamata's Theorem (see Theorem 1.5.11 in [4]). The above computation gives the desired property (4.14), which completes the proof.

\section{References}

[1] Adler, R.J. (1990). An Introduction to Continuity, Extrema, and Related Topics for General Gaussian Processes. Inst. Math. Statist. Lecture Notes - Monograph Series, Vol. 12, Inst. Math. Statist., Hayward CA.

[2] Berman, S.M. (1985). An asymptotic formula for the distribution of the maximum of Gaussian process with stationary increments. Journal of Applied Probability 22, 454-460.

[3] Billingsley, P. (1968). Convergence of Probability Measures. Wiley, New York.

[4] Bingham, N.H., Goldie, C.M., Teugels, J.L. (1987). Regular Variation. Cambridge University Press, Cambridge.

[5] Borst, S.C., Dębicki, K., Zwart, A.P. (2002). On the superposition of Gaussian and On-Off processes. In preparation.

[6] Breiman, L. (1965). On some limit theorems similar to the arc-sin law. Theory of Probability and its Applications 10, 323-331.

[7] Cline, D., Samorodnitsky, G. (1994). Subexponentiality of the product of two random variables. Stochastic Processes and their Applications 49, 75-98.

[8] Kurtz, T. (1996). Limit theorems for workload input models. In: Kelly, F., Zachary, S., Ziedins, I. (editors). Stochastic Networks, Springer.

[9] Piterbarg, V.I. (1996). Asymptotic Methods in the Theory of Gaussian Processes and Fields. Translations of Mathematical Monographs, Vol. 148, AMS, Providence RI.

[10] Piterbarg, V.I., Prisyazhnyuk, V. (1978). Asymptotic behavior of the probability of a large excursion for a nonstationary Gaussian processes. Teor. Veroyatnost. i Mat. Statist. 18, 121-133.

[11] Samorodnitsky, G., Taqqu, M.S. (1994). Stable Non-Gaussian Random Processes. Chapman \& Hall, New York. 